\title{
Case Report \\ Steroid Refractory Autoimmune Haemolytic Anaemia Secondary to Sarcoidosis Successfully Treated with Rituximab and Mycophenolate Mofetil
}

\author{
Sarah Green, Erica Partridge, Edore Idedevbo, and Anton Borg \\ Warwick Hospital, Lakin Road, Warwick, Warwickshire CV34 5BW, UK \\ Correspondence should be addressed to Sarah Green; sarah.green16@nhs.net
}

Received 13 March 2016; Revised 11 June 2016; Accepted 3 July 2016

Academic Editor: Yoshihiro Matsukawa

Copyright (c) 2016 Sarah Green et al. This is an open access article distributed under the Creative Commons Attribution License, which permits unrestricted use, distribution, and reproduction in any medium, provided the original work is properly cited.

\begin{abstract}
Autoimmune haemolytic anaemia is not a well-recognised complication of sarcoidosis. We describe the case of a 30-year-old female who presented with acute warm haemolytic anaemia and widespread lymphadenopathy. Sarcoidosis was diagnosed on lymph node biopsy and further investigation. The haemolytic anaemia responded only to a high dose of steroids. Evidence regarding treatment of steroid refractory autoimmune haemolysis secondary to sarcoidosis is lacking. Based on the emergent evidence that both disorders share common immunopathogenic mechanisms involving Thl and Th17 lymphocytes, our patient was given rituximab and mycophenolate mofetil to successfully suppress the haemolysis and sarcoid activity.
\end{abstract}

\section{Introduction}

Sarcoidosis is a multisystem granulomatous disease of unknown aetiology. The evidence is suggestive of an exaggerated granulomatous reaction which occurs in genetically susceptible individuals following exposure to a yet unidentified antigen [1]. It commonly presents with lung involvement such as pulmonary fibrosis, skin lesions such as erythema nodosum, eye involvement, and lymphadenopathy. It rarely affects the heart, kidneys, nervous system, and parotid gland. Spontaneous resolution occurs over a period of 2 years in $50 \%$ of patients. Prognosis is dependent on the severity of critical organ involvement. Mortality is higher than in the general population due to sarcoidosis itself [2].

Presentation with autoimmune haemolytic anaemia is not well recognised in sarcoidosis. Autoimmune haemolytic anaemia occurs when autoantibodies target antigens on red blood cells. It affects 1 to 3 in 100,000 people per year and it can be life-threatening [3]. It may be secondary to malignant lymphoproliferative disorders, viral infections, drugs, and other autoimmune disorders. Most cases are idiopathic. The presentation of haemolysis is typically with anaemia and/or jaundice.

\section{Case Presentation}

A 30-year-old previously healthy female presented with a 2-day history of progressive dizziness, shortness of breath, jaundice, and abdominal pain. Over the last 6 months, the patient had lost 3 stones in weight which she attributed to dieting. She was not taking medications. There was no family history of anaemia, jaundice, or gallstones.

Clinically, the patient was jaundiced and had left submandibular lymphadenopathy and splenomegaly.

Initial blood tests revealed severe spherocytic haemolytic anaemia (see Table 1). A red cell enzyme deficiency screen, including G6PD, was done once the haemolysis was subdued with treatment. Flow cytometry screening for paroxysmal nocturnal haemoglobinuria and basic Coombs tests were negative. A super Coombs done later was positive for warm IgG autoantibodies. A viral screen including HIV, $\mathrm{EBV}$, and CMV and a mycoplasma serology were negative. Immunoglobulin levels were increased but the paraprotein was absent. Autoimmune and vasculitic screens, including antinuclear, anti-extractable nuclear, and anti-neutrophil cytoplasmic autoantibodies, were absent. A CT of the neck, abdomen, and pelvis showed extensive neck, mediastinal, 
TABLE 1: Blood test results on admission.

\begin{tabular}{lc}
\hline Haemoglobin & $57 \mathrm{~g} / \mathrm{L}$ \\
Reticulocytes & $163 \times 10^{9} / \mathrm{L}$ \\
White cell count & $3.01 \times 10^{9} / \mathrm{L}$ \\
Platelets & $126 \times 10^{9} / \mathrm{L}$ \\
LDH & $1899 \mathrm{IU} / \mathrm{L}$ \\
Haptoglobin & $<0.1 \mathrm{~g} / \mathrm{L}$ \\
Bilirubin & $101 \mu \mathrm{mol} / \mathrm{L}$ \\
\hline
\end{tabular}

and upper abdominal lymphadenopathy and significant splenomegaly. As lymphoma was suspected, a cervical lymph node biopsy was taken, which showed noncaseating and nonnecrotising granulomatous lymphadenitis. The ratio of CD4 : CD8 T-lymphocytes in lymph node biopsy was 5.8 on flow cytometry analysis and 4.0 on immunohistochemistry. Sarcoidosis was diagnosed on exclusion of other causes of such lymphadenitis. The bone marrow was normal other than for erythroid hyperplasia. There was no evidence of pulmonary involvement on the CT scan and pulmonary function tests. Serum angiotensin-converting enzyme (ACE) was elevated and adjusted serum calcium was borderline high. Alkaline phosphatase and 1,25-dihydroxyvitamin D levels were normal.

The patient was transfused with 4 units of blood because of dyspnoea at rest. Immunosuppressive treatment with high dose prednisolone $80 \mathrm{mg}$ daily $(1 \mathrm{mg} / \mathrm{kg} /$ day) was started and slowly reduced after 2 weeks when there was no further haemolysis and need for blood transfusion. The haemolysis recurred 4 weeks after initiation of treatment when the patient was on $40 \mathrm{mg}$ daily of prednisolone. Four pulses of rituximab $375 \mathrm{mg} / \mathrm{m}^{2}$ and azathioprine $1 \mathrm{mg} / \mathrm{kg} /$ daily were then given. Despite normal thiopurine methyltransferase (TMT) enzyme levels, azathioprine caused severe neutropenia and was stopped after 2 weeks. Mycophenolate mofetil (MMF) 1 gram twice daily was started as an alternative. Over a period of 6 weeks, the haemolysis subsided and the patient became transfusion independent with normal haemoglobin levels. A CT scan done 3 months after starting MMF showed that the lymphadenopathy and splenomegaly had decreased significantly. ACE and calcium levels had normalised. The dose of steroids was tailed off 20 weeks after presentation.

\section{Discussion}

There are few reports of sarcoidosis associated with autoimmune haemolytic anaemia. In the literature, it was unclear if the association was causal or coincidental. In 1954, a case report and literature review identified 5 cases [4]. Since then, a few reports of associations between haemolytic anaemia and various types of sarcoidosis have been published [5-13]. Taken together, the case reports indicate no clear correlation between the stage of sarcoidosis and haemolytic anaemia or a poorer outcome. Haemolytic anaemia was the initial presentation in 3 of the published reports, as in our case [57]. Of the patients who presented with haemolytic anaemia, the underlying sarcoidosis varied from stage 0 [8] to stage 3
[6]. Others with a known diagnosis of sarcoidosis developed haemolytic anaemia later in the disease $[9,10]$. In one of the case reports, the haemolysis was fatal despite treatment [11].

Evidence is poor in the literature as to the best treatments of autoimmune haemolytic anaemia and sarcoidosis. Treatment of haemolysis includes that of the cause, as in our case report. Blood transfusion is often required in severe acute cases. Transfusion may be problematic because the donor red blood cells are difficult to crossmatch and are consumed rapidly. A recent literature review recommends steroids as first line treatment for warm autoimmune haemolytic anaemia [14]. Rituximab is the preferred second line treatment; it is effective in $70-80 \%$ of cases. Another option is splenectomy in refractory cases. Other options to be considered include immunosuppressant drugs such as azathioprine and cyclophosphamide. Other treatments such as immunoglobulins and danazol have little evidence of success. High dose cyclophosphamide and alemtuzumab have been used in severe refractory cases. For cold autoimmune haemolytic anaemia, rituximab is first line.

Effective treatment of autoimmune haemolysis in sarcoid patients is dependent on a better understanding of the pathophysiological link between sarcoidosis and haemolytic anaemia. Patients with sarcoidosis have clusters of CD4+ T-helper Th1 lymphocytes in affected organs [15]. More recently, CD4+ T-helper Th17 cells were implicated in the formation of sarcoid granulomas [16]. It was hypothesised that Th1 cells are responsible for the initial autoantibody response and that the Th17 cells drive the chronic inflammation in autoimmune conditions including sarcoidosis and autoimmune haemolytic anaemia [17]. These shared immunopathogenic processes may explain why sarcoidosis and haemolytic anaemia occur together in the same patient and offer a therapeutic strategy.

Rituximab reduces the local Th17 response, which is associated with reduced inflammation and a better clinical outcome in rheumatoid patients. The inhibition of the Th17 response by rituximab was dependent on B-lymphocyte depletion [18]. A similar mechanism may apply to haemolytic anaemia and sarcoidosis. Rituximab also reduces autoantibodies to red cells by depleting the B cells. Most clinical trials have used a 4 weekly dose of $375 \mathrm{mg} / \mathrm{m}^{2}$. A meta-analysis of 21 studies found an overall response rate of $73 \%$ and a complete response rate of $42 \%$ [19]. Out of 364 patients, 22 had severe adverse events. It has been suggested that rituximab is a safer second line treatment, when compared to splenectomy.

In view that a response to rituximab often takes 8 to 16 weeks, MMF was also used in our case in order to obtain quicker immunosuppression and thus an earlier reduction of the high steroid doses. MMF has been shown to suppress both Th1 and Th17 cell subsets in several autoimmune and inflammatory disorders. Whilst MMF appears to offer no extra benefit to sarcoidosis patients compared to more established steroid-sparing agents, it was beneficial in our patient who was intolerant to azathioprine. As yet there are no studies that test the efficacy of MMF as the initial steroidsparing agent in sarcoidosis [20].

It is not yet clear how rituximab and MMF work synergistically. The combination has been shown to be effective in 
a case of refractory autoimmune haemolytic anaemia but not related to sarcoidosis [21]. MMF inhibits the proliferation of $\mathrm{T}$ - and B-lymphocytes. The reason for both agents working so well together could be the impact on Th17 cells. As previously noted, rituximab reduces the Th17 response [18]. MMF has also been found to have a strong inhibitory effect on the Th17 cell related immune response, which could explain why the combination of rituximab and MMF is successful in treating refractory sarcoidosis and haemolytic anaemia [22].

Sarcoidosis does not always require treatment. Between 20 and $70 \%$ of patients require systemic therapy if they are at risk from or have already suffered organ damage [1]. There have been 13 trials of steroid treatment and 5 trials of immunosuppressive and cytotoxic drugs. In all these studies, there was no standard protocol for the dose and duration of steroid treatment. This may be because of the high interindividual variability in response to steroids and other immunosuppressants. Prednisolone is frequently given at a dose of 20-40 mg for 6-12 weeks. The dose is usually reduced after this. Patients often receive treatment for 12 months to prevent relapse, but it has been suggested that treatment should be stopped after 6 months. Depending on the response to treatment, it may need to be continued for several years. Due to the side effects of steroids, alternative steroid-sparing options should be considered for long-term treatment.

Although there is no established treatment for sarcoidosis complicated with haemolytic anaemia, current practice involves similar treatments for both conditions. Several of the published cases have had a good response to steroids and no further relapse at the time of being published, although their long-term follow-up was not described [5-7, 12]. In another report, the patient was treated with steroids and danazol [13]. A patient who had a splenectomy had a relapse and required long-term steroid treatment [4]. Another patient who had a splenectomy had an initial recovery but died following a haemolytic crisis one year later [11].

Sarcoidosis and autoimmune haemolytic anaemia may have a similar pathological basis involving Th1 and Th17 lymphocytes. There are no guidelines on how to treat sarcoidosis with steroid refractory haemolytic anaemia because the evidence base for this is very poor. Our patient is the first documented case of sarcoidosis complicated with haemolytic anaemia to be treated successfully with rituximab and immunosuppressive bridging with MMF. Studies based on novel immunotherapeutic strategies, including Blymphocyte depletion, to modulate the phenotype of Th17 cells, particularly away from the Th1 phenotype, should be considered.

\section{Competing Interests}

The authors declare that there are no competing interests regarding the publication of this paper.

\section{Acknowledgments}

Thanks are due to the haematology research fund at Warwick Hospital.

\section{References}

[1] D. Valeyre, A. Prasse, H. Nunes, Y. Uzunhan, P.-Y. Brillet, and J. Müller-Quernheim, "Sarcoidosis," The Lancet, vol. 383, no. 9923, pp. 1155-1167, 2014.

[2] J. J. Swigris, A. L. Olson, T. J. Huie et al., "Sarcoidosis-related mortality in the United States from 1988 to 2007," American Journal of Respiratory and Critical Care Medicine, vol. 183, no. 11, pp. 1524-1530, 2011.

[3] G. F. Bass, E. T. Tuscano, and J. M. Tuscano, "Diagnosis and classification of autoimmune hemolytic anemia," Autoimmunity Reviews, vol. 13, no. 4-5, pp. 560-564, 2014.

[4] A. E. Davis, J. P. Belber, and E. R. Movitt, "The association of hemolytic anemia with sarcoidosis," Blood, vol. 9, no. 4, pp. 379383, 1954

[5] N. Yasuda, M. Kohda, M. Nomura et al., "Sarcoidosis in a patient with autoimmune hemolytic anemia," Nihon Kyobu Shikkan Gakkai Zasshi, vol. 34, no. 8, pp. 931-936, 1996.

[6] R. K. Ghaddar and T. H. Muzaffar, "Pulmonary sarcoidosis and autoimmune hemolytic anemia: possible common immune pathogenesis," Archives of Iranian Medicine, vol. 14, no. 2, pp. 146-148, 2011.

[7] K. Chernow, T. Donegan, T. Milman, and D. Henry, "Sarcoidosis, complete heart block, and warm autoimmune hemolytic anemia in a young woman," The Journal of Community and Supportive Oncology, vol. 13, no. 4, pp. 159-161, 2015.

[8] B. Desablens, A. Pruna, M. F. Gontier, and J. Messerschmitt, "Autoimmune haemolytic anaemia associated with bone marrow sarcoidosis," Acta Haematologica, vol. 71, no. 3, pp. 204206, 1984.

[9] S. Wyss and C. Maier, "Hemolytic anemia in sarcoidosis of the spleen," Acta Haematologica, vol. 37, no. 2, pp. 126-136, 1967.

[10] M. Meregalli, R. Bosisio, C. L. Delpiano, and M. Spinelli, "Monolateral sarcoidosis of parotid gland associated with hemolytic anemia: description of a case diagnosed by cytohistology," Haematologica, vol. 76, no. 4, pp. 321-323, 1991.

[11] U. Thadani, C. P. Aber, and J. J. Taylor, "Massive splenomegaly, pancytopenia and haemolytic anaemia in sarcoidosis," Acta Haematologica, vol. 53, no. 4, pp. 230-240, 1975.

[12] P. Semple, "Thrombocytopenia, haemolytic anaemia and sarcoidosis," British Medical Journal, vol. 4, no. 5994, pp. 440-441, 1975.

[13] H. Kondo, S. Sakai, and Y. Sakai, "Autoimmune haemolytic anaemia, Sjogren's syndrome and idiopathic thrombocytopenic purpura in a patient with sarcoidosis," Acta Haematologica, vol. 89, no. 4, pp. 209-212, 1993.

[14] W. Barcellini, "Current treatment strategies in autoimmune hemolytic disorders," Expert Review of Hematology, vol. 8, no. 5, pp. 681-691, 2015.

[15] R. P. Baughman, E. E. Lower, and R. M. du Bois, "Sarcoidosis," The Lancet, vol. 361, no. 9363, pp. 1111-1118, 2003.

[16] M. Facco, A. Cabrelle, A. Teramo et al., "Sarcoidosis is a Th1/Th17 multisystem disorder," Thorax, vol. 66, no. 2, pp. 144150, 2011.

[17] K. K. Hoyer, W. F. Kuswanto, E. Gallo, and A. K. Abbas, "Distinct roles of helper T-cell subsets in a systemic autoimmune disease," Blood, vol. 113, no. 2, pp. 389-395, 2009.

[18] F. L. van de Veerdonk, B. Lauwerys, R. J. Marijnissen et al., "The anti-CD20 antibody rituximab reduces the Th17 cell response," Arthritis \& Rheumatism, vol. 63, no. 6, pp. 1507-1516, 2011. 
[19] Q. Reynaud, I. Durieu, M. Dutertre et al., "Efficacy and safety of rituximab in auto-immune hemolytic anemia: a meta-analysis of 21 studies," Autoimmunity Reviews, vol. 14, no. 4, pp. 304-313, 2015.

[20] N. Hamzeh, A. Voelker, A. Forssén et al., "Efficacy of mycophenolate mofetil in sarcoidosis," Respiratory Medicine, vol. 108, no. 11, pp. 1663-1669, 2014.

[21] N. O'Connell, M. Goodyer, M. Gleeson et al., "Successful treatment with rituximab and mycophenolate mofetil of refractory autoimmune hemolytic anemia post-hematopoietic stem cell transplant for dyskeratosis congenita due to TINF2 mutation," Pediatric Transplantation, vol. 18, no. 1, pp. E22-E24, 2014.

[22] F. Abadja, S. Atemkeng, E. Alamartine, F. Berthoux, and C. Mariat, "Impact of mycophenolic acid and tacrolimus on Th17related immune response," Transplantation, vol. 92, no. 4, pp. 396-403, 2011. 


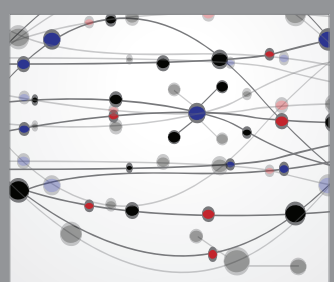

The Scientific World Journal
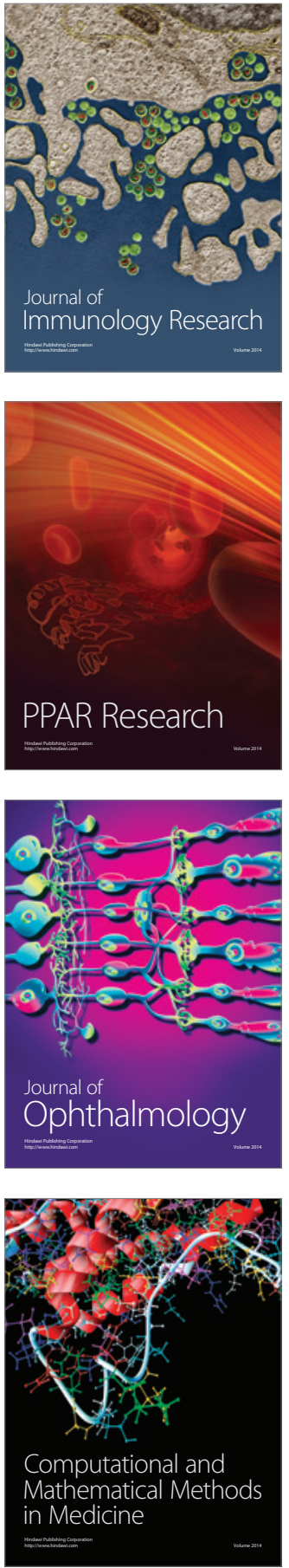

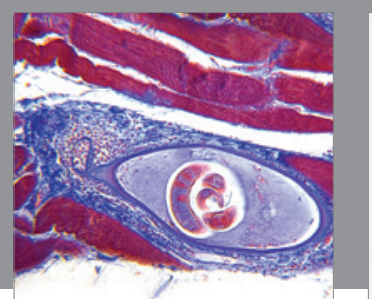

Gastroenterology Research and Practice

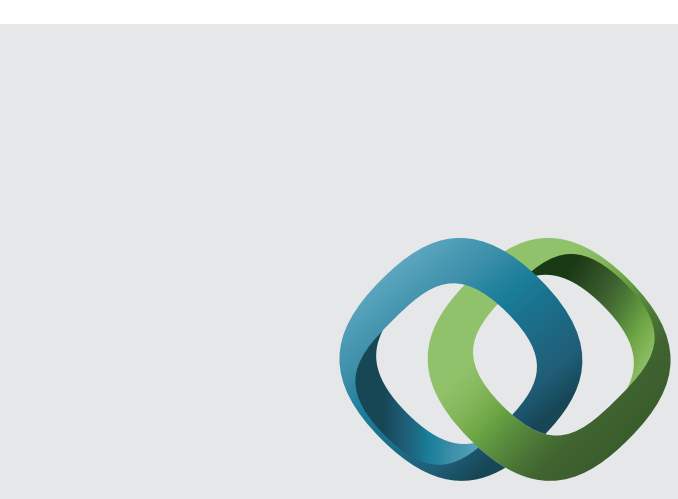

\section{Hindawi}

Submit your manuscripts at

http://www.hindawi.com
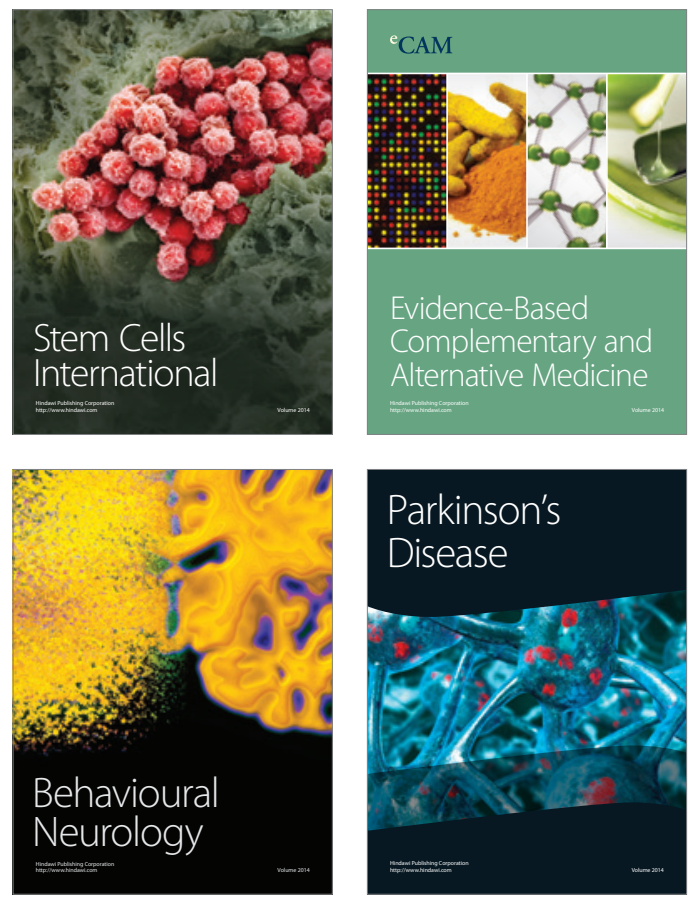
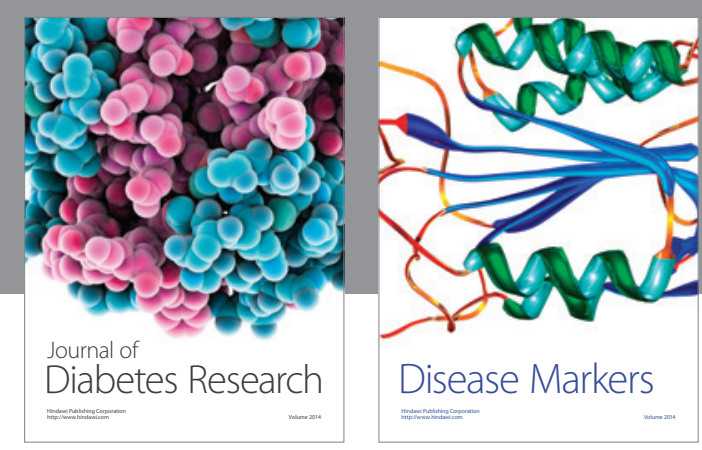

Disease Markers
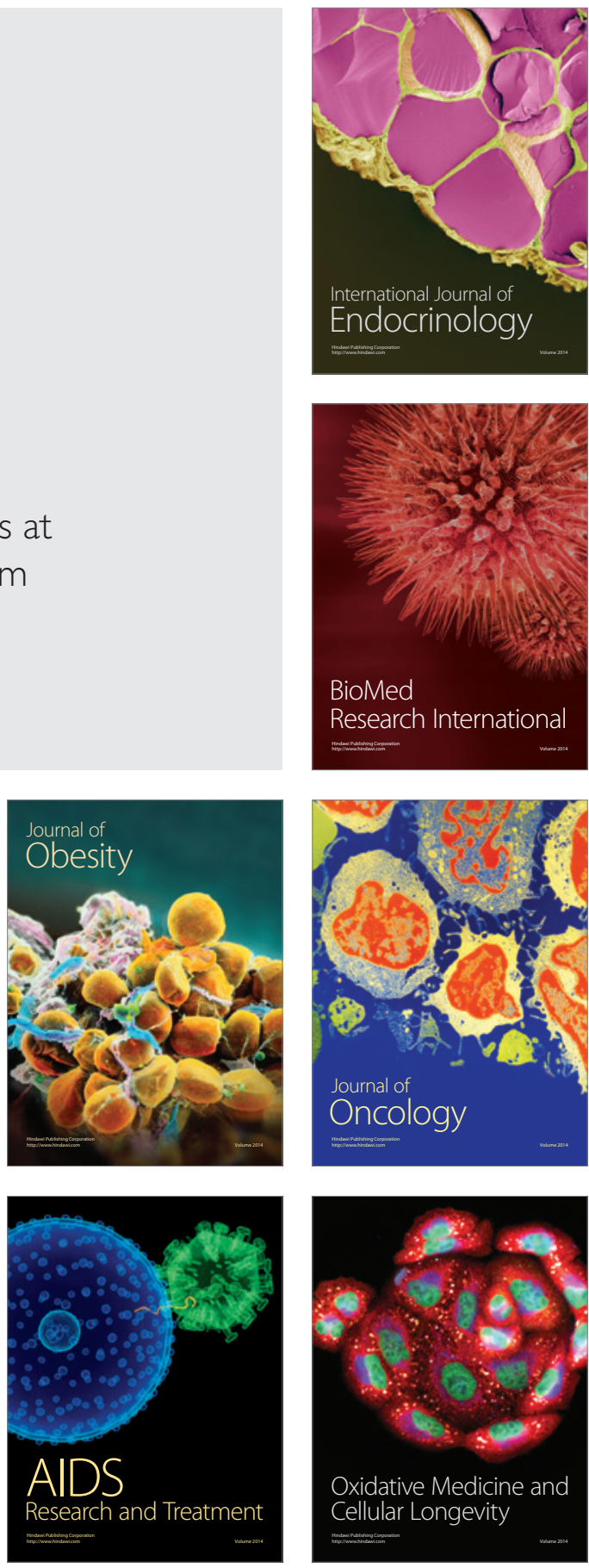